\title{
Therapeutic Roles of Bile Acid Signaling in Chronic Liver Diseases
}

\author{
Yan Li and Lun-Gen Lu* \\ Department of Gastroenterology, Shanghai General Hospital, Shanghai Jiaotong University School of Medicine, Shanghai, China
}

\begin{abstract}
Bile acids (BAs) are the major metabolic product of cholesterol, having detergent-like activities and being responsible for absorption of lipid and lipid-soluble vitamins. In addition, it has been increasingly recognized that BAs are important signaling molecules, regulating energy metabolism and immunity. Under physiological circumstances, synthesis and transport of BAs are precisely regulated to maintain bile acid homeostasis. Disruption of bile acid homeostasis results in pathological cholestasis and metabolic liver diseases. During the last decades, BAs have been gradually recognized as an important therapeutic target for novel treatment in chronic liver diseases. This review will provide an update on the current understanding of synthesis, transport and regulation of BAs, with a focus on the therapeutic roles of bile acid signaling in chronic liver diseases.
\end{abstract}

Citation of this article: Li Y, Lu LG. Therapeutic roles of bile acid signaling in chronic liver diseases. J Clin Transl Hepatol 2018;6(4):425-430. doi: 10.14218/JCTH.2018.00025.

\section{Introduction}

Bile acids (BAs) are the primary end metabolic product of cholesterol, having detergent-like activities and playing important roles in lipid absorption and utilization of lipids.

Keywords: BAs; Cholestasis; Chronic liver diseases; FXR; TGR5.

Abbreviations: 3'-UTR, 3' untranslated region; 5'-UTR, 5' untranslated region; ALD, alcoholic liver disease; ASBT, apical sodium-dependent BA transporter; BAs, bile acids; BACS, bile acid:CoA synthase; BAT, bile acid:amino acid transferase; BSEP, bile salt export pump; CA, cholic acid; CDCA, chenodeoxycholic acid; CYP27A1, cytochrome P450 27A1; CYP7A1, cytochrome P450 7A1; CYP7B1, cytochrome P450 7B1; CYP8B1, cytochrome P450 8B1; DBD, DNA binding domain; DCA, deoxycholic acid; FDA, US Food and Drug Administration; FGF15, fibroblast growth factor 15; FXR, farnesoid X receptor; GLP-1, glucagon-like peptide-1; HNF- $4 \alpha$, hepatocyte nuclear factor- $4 \alpha$; IBABP, ileal bile acid binding protein; LBD, ligand binding domain; LCA, lithocholic acid; LRH-1, liver-related homologue-1; MDR3, phospholipid export pump; MRP2, canalicular conjugate export pump; NAFLD, nonalcoholic fatty liver disease; NASH, nonalcoholic steatohepatitis; norUDCA, nor-ursodeoxycholic acid; NTCP, Na+/taurocholate cotransporting polypeptide; OATP2, organic anion transporting proteins; OCA, obeticholic acid; OST $\alpha / \beta$, organic solute transporter; PBC, primary biliary cholangitis; PCSK9, proprotein convertase subtilisin/kexin type-9; PKA, protein kinase A; PPAR $\alpha$, peroxisome proliferator-activated receptor $\alpha$; PSC, primary sclerosing cholangitis; PXR, pregnane $X$ receptor; $\mathrm{RXR}$, retinoid $\mathrm{X}$ receptor; $\mathrm{SHP}$, small heterodimer partner; $T A D$, transactivating domain; TGR5, transmembrane $G$ protein coupled receptor 5; TSH, thyroid-stimulating hormone; UDCA, ursodeoxycholic acid; VDR, vitamin $D$ receptor.

Received: 10 April 2018; Revised: 22 August 2018; Accepted: 24 August 2018 *Correspondence to: Lun-Gen Lu, Department of Gastroenterology, Shanghai General Hospital, Shanghai Jiaotong University School of Medicine, 100 Haining Road, Shanghai 200080, China. Tel: +86-21-63240090, Fax: +86-21-63241377, E-mail: lungenlu1965@163.com
As natural detergents, BAs might disrupt lipid components of cell membranes and advocate oxidative damage. Hence, accumulation of BAs within hepatocytes actually leads to apoptosis or necrosis of hepatocytes, which is principally responsible for cholestatic liver damage. ${ }^{1}$ Besides, BAs are also natural ligands for several nuclear receptors and membrane receptors, including farnesoid $X$ receptor (FXR), pregnane $X$ receptor (PXR), vitamin $D$ receptor (VDR) and transmembrane $G$ protein coupled receptor 5 (TGR5). BAs are important signaling molecules as well, playing critical roles in regulation of multiple metabolic pathways, including lipid, glucose, drug and energy metabolism. Additionally, BAs play a vital role in gut bacteria homeostasis.

BAs have been considered as important therapeutic targets for treatment of cholestatic and metabolic liver diseases. ${ }^{2}$ Cholestatic liver diseases are currently associated with impaired bile flow and accumulation of BAs within the liver, resulting in hepatic inflammation and liver injury. Chronic cholestasis increases the risk of hepatocellular carcinoma and cholangiocarcinoma. And, it has been illustrated that FXR and BAs play important roles in both alcoholic liver disease (ALD) and nonalcoholic fatty liver disease (NAFLD). ${ }^{3,4}$ The emerging therapeutic roles of bile acid signaling in chronic liver diseases are discussed in this review.

\section{Synthesis and transport of BAs}

BAs are principal components of the bile, which mainly contains bilirubin, cholesterol, phospholipids, electrolytes, and water. Synthesis, transport and pool size of the BAs are precisely regulated under physiological conditions. As the predominant end product of cholesterol catabolism, synthesis of BAs is a complex process within hepatocytes. A series of the cytochrome P450 subfamily members are involved in the process of BAs production. There are two distinct pathways responsible for primary BAs production.

The classical pathway produces cholic acid (CA) and chenodeoxycholic acid (CDCA), which accounts for approximately $90 \%$ of primary BAs synthesis in humans. Cytochrome P450 7A1 (CYP7A1) and cytochrome P450 8B1 (CYP8B1) are the key enzymes in the classical pathway of BAs synthesis. The alternative pathway produces CDCA predominantly, which accounts for about $10 \%$ of primary BAs synthesis in humans. In the alternative pathway, cytochrome P450 27A1 (CYP27A1) and cytochrome P450 7B1 (CYP7B1) are responsible for hydroxylation and oxidation of cholesterol. The different contributions of the two pathways determine the internal composition of the BAs. ${ }^{5}$

BAs conjugated with glycine or taurine are known as bile salts, which are more hydrophilic, facilitating secretion into 
the bile. Moreover, bile salts are generally considered to be less toxic. Bile acid:CoA synthase (BACS) and bile acid:amino acid transferase (BAT) are required in this procedure. ${ }^{6}$ Conjugated BAs are then secreted via the bile salt export pump (BSEP) into the canaliculi. Secreted primary BAs gradually flow through the canaliculi, interlobular bile ducts, left/right hepatic duct, and common hepatic duct, eventually reaching the gallbladder. BAs are currently stored and concentrated in gallbladder. The concentrated bile in gallbladder flows into the intestinal lumen through the major duodenal papilla after food intake.

In the intestine, primary BAs are metabolized by gut bacteria, producing secondary BAs, mainly including deoxycholic acid (DCA), lithocholic acid (LCA) and ursodeoxycholic acid (UDCA). Both primary and secondary BAs comprise the total BAs pool, with a relatively fixed proportion. Alteration of the size and composition of the BAs pool might accelerate pathological processes, such as cholestasis and cholelithiasis.

BAs are amphipathic molecules with detergent-like actions, which are responsible for their roles in facilitating absorption of lipid and lipid-soluble vitamins in the intestine. After their physiological functions are completed in the intestine, the BAs reach the distal ileum, where $95 \%$ of BAs are reabsorbed via a sodium-dependent process. The apical sodium-dependent BA transporter (ASBT) expressed in the brush border membrane of the distal ileum is the major transporter mediating the reabsorption of BAs. ASBT mediates the active transfer of BAs across the luminal plasma membrane to the ileal bile acid binding protein (IBABP), which facilitates intracellular diffusion of BAs to the basolateral membrane. The organic solute transporter $(\mathrm{OST} \alpha / \beta)$ mediates the exit of BAs across the basolateral plasma membrane, and BAs then enter the portal bloodstream.

Circulatory BAs are taken up by the $\mathrm{Na}+$ /taurocholate cotransporting polypeptide (NTCP; for conjugated BAs) and organic anion transporting proteins (OATP2s; for the unconjugated BAs) at the basolateral membrane of hepatocytes, and are then exported into the bile by BSEP as well as the canalicular conjugate export pump (MRP2). MRP2 also mediates excretion of bilirubin. The phospholipid export pump (MDR3) mediates excretion of phosphatidylcholine, forming mixed micelles together with BAs and cholesterol in bile. ${ }^{7,8}$

This efficient cycle between intestine and liver is known as enterohepatic circulation. It is repeated several times in a day, ensuring $95 \%$ of BAs are reabsorbed and returned to the BAs pool, leaving only $5 \%$ of the total BAs pool in the intestinal lumen and to be finally excreted in feces. The liver produces approximately $0.2 \mathrm{~g}-0.6 \mathrm{~g}$ BAs daily, as a supplement for the BAs pool, maintaining bile acid homeostasis. It should be emphasized that the human BAs pool mainly consists of hydrophobic bile acid, including chenodeoxycholic acid (CDCA), cholic acid (CA), and DCA. Approximately 95\% of $\mathrm{BAs}$ are reabsorbed in the gut and transported back to the liver, whereas most of the LCA is excreted in feces. Only a small portion of LCA circulated to the liver is sulfo-conjugated by sulfotransferase and eventually secreted into bile. ${ }^{9}$

\section{Regulation of BAs synthesis and metabolism}

Under physiological circumstances, the synthesis of BAs is precisely regulated by a complicated net of multiple signaling pathways. Regulation of CYP7A1 transcription, the rate-limiting enzyme of BAs synthesis, is the most critical step of several pathways. The enterohepatic circulation of BAs plays a vital role in feedback inhibition of BAs synthesis. Circadian rhythm, food intake, thyroid function, as well as gut microbiota play important roles in regulation of BAs synthesis.

As mentioned above, both CYP7A1 and CYP8B1 are required for the classic pathway of BAs synthesis, which produces the majority of bile acid in humans. CYP7A1 is currently considered to be the rate-limiting enzyme in BAs synthesis. Thus, regulation of CYP7A1 mRNA transcription is considered as a critical step in regulation of BAs synthesis. BAs returning to the liver via enterohepatic circulation could inhibit CYP7A1 transcription. The $3^{\prime}$ untranslated region (3'-UTR) of CYP7A1 mRNA has an extremely short half-life (30 min). It has been elucidated that BAs reduce CYP7A1 mRNA stability and promote degradation of CYP7A1 mRNA via the bile acid response elements located in the $3^{\prime}-$ UTR. ${ }^{10}$ Besides, BAs as well inhibit CYP7A1 mRNA transcription by targeting its $5^{\prime}$ untranslated region ( $5^{\prime}$-UTR) and inhibit transcriptional activities of the promoter. ${ }^{11,12}$

There are two FXR-mediated pathways for bile acid inhibition of CYP7A1 gene transcription. In the liver, BAs bind to FXR and induce expression of small heterodimer partner (SHP). SHP works as a corepressor, inhibiting transcriptional activity of hepatocyte nuclear factor- $4 \alpha$ (HNF- $4 \alpha)$ and liverrelated homologue-1 (LRH-1), which subsequently downregulate transcription of CYP7A1 and CYP8B1. ${ }^{13}$ In the intestine, BAs bind to FXR and then induce fibroblast growth factor 15 (FGF15), which is secreted into the portal bloodstream and finally activates the hepatic FGFR4/ $\beta$-Klotho complex. The activated complex then inhibits CYP7A1 transcription via JNK and ERK1/2MAPK pathways. ${ }^{14,15}$

FXR also stimulates bile acid conjugation by up-regulation of BACS/BAT transcription, which greatly affects the composition of the BAs pool. Furthermore, FXR binds to the BSEP gene promoter and induces the expression of BSEP in the canalicular membrane, facilitating bile acid secretion. Thus, it is concluded that FXR is of vital importance in the regulation of BAs synthesis, enterohepatic circulation and internal composition of the BAs pool. Currently, FXR deficiency is known to result in cholestatic diseases.

Food intake, glucose metabolism and circadian rhythms are involved in regulation of BAs synthesis as well. Li et al. ${ }^{16}$ suggested that insulin at physiological concentrations rapidly stimulates CYP7A1 expression, whereas elevated serum insulin level in the insulin resistance state activates the steroid response element binding protein-1c, which inhibits CYP7A1 mRNA transcription. Glucagon and CAMP are thought to inhibit CYP7A1 expression in human hepatocytes via activation of protein kinase $A$ (PKA) and phosphorylation of HNF-4 $\alpha .{ }^{17}$ BAs synthesis increases in the morning despite food intake, due to increased CYP7A1 expression in the morning. The circadian rhythms of CYP7A1 expression might be associated with the orphan nuclear receptor Reverb $\alpha$ and the D-site binding protein. ${ }^{18}$

The gut microbiota regulates BAs synthesis and metabolism. The gut microbiota is responsible for deconjugating BAs and converting primary BAs into secondary BAs. ${ }^{19}$ In humans, Lactobacillus, Bifidobacteria, Enterobacter, Bacteroides, and Clostridium are mainly responsible for BAs metabolism in the gut. It has been suggested that altering the intestinal microbiota evoked by any influencing factors would subsequently alter the composition of intestinal BAs. ${ }^{20}$ In healthy human beings, cholesterol is primarily catalyzed to CA and CDCA via the classical bile acid synthetic pathway. 


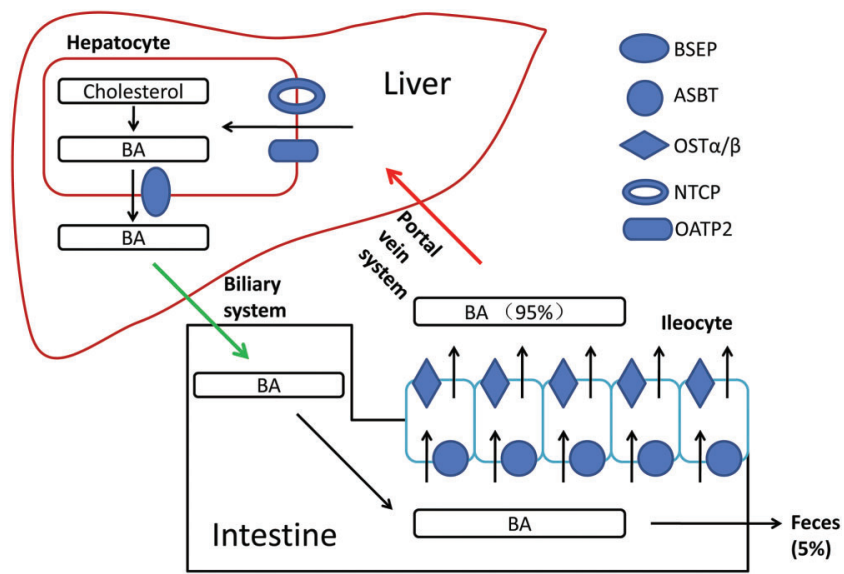

Fig. 1. Enterohepatic circulation. Synthesis of bile acids (BAs) occur within hepatocytes and BAs are secreted into bile via bile salt export pump (BSEP). 95\% of the bile acid pool are re-absorbed in intestine. Apical sodium-dependent BA transporter (ASBT) expressed in the brush border membrane of the distal ileum mediates bile acids crossing the luminal plasma membrane into ileocytes. Organic solute transporter $(\mathrm{OST} \alpha / \beta)$ mediates bile acid exiting across the basolateral plasma membrane into portal bloodstream. Circulatory bile acids are taken up by taurocholate cotransporting polypeptide (NTCP) and organic anion transporting proteins (OATP2) at the basolateral membrane of hepatocytes, and then exported into bile by BSEP.

Bile acid $7 \alpha$-dehydroxylating bacteria are abundant, maintaining the normal composition of BAs pool.

Bile salts secreted into the small intestine are able to prevent dysbiosis and proinflammatory cytokines release. In cirrhotic patients, up-regulated proinflammatory cytokines' release inhibits CYP7A1 transcription and inhibits the classical pathway. The alternative pathway then becomes the main pathway for BAs synthesis. Patients with hepatic cirrhosis generally have a lower abundance of bacteria with $7 \alpha$-dehydroxylating activity, and the ratios of DCA/CA and LCA/CDCA significantly decrease. Different contributions of the two distinct BA synthetic pathways and dysbiosis lead to alteration of the BAs pool composition. Whereas, BAs in turn have direct effect on the gut microbiota. ${ }^{21,22}$

Thyroid function is another important factor regulating BAs synthesis. Cholestatic changes currently occur in patients with hypothyroidism. It has been confirmed that thyroid hormone reduces proprotein convertase subtilisin/kexin type-9 (PCSK9) and stimulates BAs synthesis in humans. ${ }^{23}$ Song et al. ${ }^{24}$ suggested that thyroid-stimulating hormone (TSH) represses BAs synthesis via a SREBP-2/HNF-4 $\alpha$ / CYP7A1 signaling pathway, independent of thyroid hormones.

Therapeutic roles of BAs signaling in chronic liver diseases

\section{UDCA and UDCA derivatives}

During the last decades, UDCA has been therapeutically used in a number of cholestatic liver diseases and metabolic liver diseases. UDCA is the most hydrophilic constituent of natural BAs. It is suggested that UDCA conjugates have antiapoptotic and antiinflammatory effects in cholestatic liver diseases and stimulate bile acid secretion. UDCA also stimulates biliary $\mathrm{HCO}_{3}$ secretion in hepatocytes and cholangiocytes, leading to stabilization of the 'biliary $\mathrm{HCO}_{3}$ umbrella', the integrated line of defense against toxic BAs. ${ }^{25,26}$

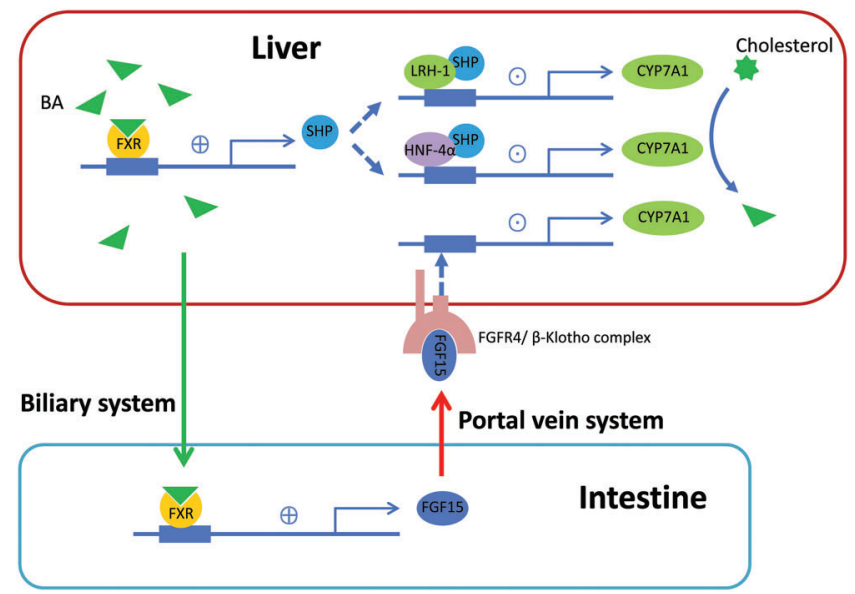

Fig. 2. Feedback regulation of bile acid (BA) synthesis through farnesoid $\mathbf{X}$ receptor (FXR). Cytochrome P450 7A1 (CYP7A1) is the rate-limiting enzyme of BA synthesis. In the liver, BA binds to FXR to induce expression of small heterodimer partner (SHP), which inhibits transcriptional activity of hepatocyte nuclear factor $4 \alpha$ (HNF- $4 \alpha$ ) as well as liver-related homologue-1 (LRH-1), and down-regulates CYP7A1 transcription. In the intestine, BA binds to FXR to induce fibroblast growth factor 15 (FGF15), which is secreted into portal bloodstream and finally activates hepatic FGFR4/ $\beta$-Klotho complex, initiating a regulatory cascade to repress CYP7A1 transcription.

At present, UDCA is the only drug approved by the US Food and Drug Administration (FDA) for primary biliary cholangitis $(P B C)$. It is reported that UDCA might improve liver functions and delay the progression of PBC. ${ }^{27}$ Combination of corticosteroids might strengthen the therapeutic efficacy of UDCA and alleviate adverse effects. ${ }^{28}$ Bosch et al. ${ }^{29}$ suggested that preventive administration of UDCA after liver transplantation for PBC patients reduces risk of recurrence. UDCA, as well, helps improve liver functions but has less benefit in improving prognosis for patients with primary sclerosing cholangitis (PSC). ${ }^{30,31}$

Furthermore, UDCA has recently emerged as a promising therapeutic medication for polycystic liver diseases. It has been confirmed that UDCA inhibits hepatic cytogenesis in experimental models of polycystic liver disease and improves symptoms in patients. ${ }^{32,33}$ However, up to $40 \%$ of PBC patients are nonresponders to UDCA therapy, who have poor prognosis despite full-dose UDCA medication. Hence, novel therapeutic approaches are required regarding UDCA nonresponders and the limited efficacy of UDCA in cholestatic disorders other than PBC.

Nor-ursodeoxycholic acid (norUDCA) is a side chainshortened homologue of UDCA with resistance to amidation, which enables its cholehepatic shunting. Non-amidated norUDCA enables 'ductular targeting' with cholangio-protective effects regarding its antiinflammatory, antilipotoxic, antifibrotic, and antiproliferative effect. NorUDCA, as well, influences lipotoxic fatty acid composition and promotes detoxification and elimination of BAs. It has been confirmed that norUDCA has therapeutic efficacy in diverse cholestatic diseases, including PSC, S. mansoni infection-induced liver fibrosis and even bile duct ligation-induced cholemic nephropathy. ${ }^{34,35}$ It has also been reported that norUDCA significantly improved liver biochemistry, in a dose-dependent manner, in PSC patients during a 12-week treatment and showed less toxicity compared to UDCA. ${ }^{36-38}$ 


\section{FXR}

FXR was first identified in 1995 as an orphan nuclear receptor. It is involved in a variety of physiological processes, including development, differentiation and metabolism. The hydrophobic BAs, as well as farnesol, are natural ligands of FXR. CDCA is the most efficacious endogenous FXR ligand, whereas hydrophilic BAs, such as UDCA and muricholic acids, do not activate FXR. Similar to most nuclear receptors, FXR consists of a transactivating domain (TAD), DNA binding domain (DBD), helix, and ligand binding domain (LBD). Corresponding ligands bind to LBD of FXR, forming a heterodimer with retinoid $X$ receptor ( $R X R$ ) and accompanied by DNA conformational changes. DBD is then exposed and actually binds to the cis-regulatory element located in the targeted gene promoters, regulating transcription of targeted gene.

As noted above, FXR down-regulates CYP7A1 gene transcription via the SHP/HNF-4 $\alpha / \mathrm{LRH}-1$ pathway and the FGFR4/ $\beta$-/JNK/ERK pathway. FXR activation down-regulates NTCP and ASBT transcription, whereas it up-regulates BSEP, BACS/ BAT, OST $\alpha / \beta$ as well as IBABP transcription. FXR activation also prevents BAs toxicity by up-regulating detoxification enzymes. ${ }^{39}$ Hence, FXR plays a key role in the regulation of BAs synthesis, excretion, transport and metabolism, and it is a prospective therapeutic target in cholestatic and metabolic liver diseases.

The semisynthetic BA derivative obeticholic acid (OCA) is a selective FXR agonist, which is considered as a prospective therapeutic agent in nonalcoholic steatohepatitis (NASH) and has recently been approved by the FDA. ${ }^{40}$ It has been confirmed that administration of OCA increases insulin sensitivity, and alleviates liver inflammation and fibrosis in patients with NAFLD. ${ }^{41}$ Coadministration of a glucagon-like peptide-1 receptor agonist IP118 with OCA might enhance the therapeutic efficacy. ${ }^{42}$ Besides, it has been demonstrated that OCA might reduce hepatic inflammation and fibrosis in cirrhosis and improve portal hypertension. ${ }^{43,44}$ A recent study showed that the nonsteroidal FXR agonist PX20606 ameliorates portal hypertension by targeting vascular remodeling and sinusoidal dysfunction. ${ }^{44}$

BAR502 is a dual FXR and GPBAR1 agonist, which protects against liver damage caused by high-fat diet by promoting the browning of adipose tissue. ${ }^{45}$ Another study demonstrates that 3-deoxy-6-ethyl derivative of CDCA (BAR704) might reduce liver fibrosis by interfering with the TGF- $\beta$ /SMAD3 pathway in hepatic stellate cells. In short, FXR is a prospective therapeutic target and requires further studies in the near future.

\section{PPAR $\alpha$}

Peroxisome proliferator-activated receptor $\alpha$ (PPAR $\alpha)$ is a member of peroxisome proliferator-activated receptor subfamily. It was first identified in 1990 and thought to be engaged in multiple physiological processes. PPAR $\alpha$ activation shows antiinflammatory effect, due to its transcriptional regulating activities. PPAR activation increases MDR3 expression in the canalicular membrane, which facilitates phosphatidylcholine secretion and protects cholangiocytes against bile acid toxicity. Besides, it inhibits CYP7A1 transcription. ${ }^{46}$

Fibrates are PPAR $\alpha$ agonists, which are traditionally used in the lipid-lowering therapy. It seems that combination therapy with UDCA and fibrates in PBC patients who are incomplete UDCA responders may improve prognosis. ${ }^{4-49}$ It has been recently elucidated that fenofibrate inhibits intrahepatic cholestasis via the PPAR $\alpha$-dependent JNK signaling pathway. ${ }^{50}$ However, safety and cost-effectiveness of fibrates therapy requires further evaluation.

\section{TGR5}

TGR5 is a G protein-coupled bile acid receptor and plays a key role in the regulation of energy metabolism. It is expressed in cholangiocytes, intestinal epithelial cells, Kupffer cells, as well as in CD14+ monocytes of the peripheral blood. The most potent natural ligands are taurine-conjugated secondary BAs, such as tauro-lithocholate. TGR5 activation stimulates glucagon-like peptide-1 (GLP-1) secretion to improve insulin sensitivity and hepatic metabolism. Activation of TGR5 in biliary epithelial cells promotes chloride and bicarbonate secretion, triggers cell proliferation, and prevents apoptosis. Furthermore, TGR5 activation inhibits expression and secretion of proinflammatory cytokines, which eventually alleviates hepatic and intestinal inflammation. ${ }^{51,52}$

It has been suggested that TGR5 activation alleviates hepatic encephalopathy via reducing microglia activation/proliferation and proinflammatory cytokine production. ${ }^{53}$ INT-777 is a selective TGR5 agonist, exhibiting extensive therapeutic effect in colitis, atherosclerosis, and hepatic steatosis. ${ }^{54}$ INT767 is a dual FXR and TGR5 agonist. Rizzo et al..$^{55}$ have demonstrated the therapeutic effect of INT-767 in hepatic steatosis. However, TGR5 activation might also accelerate proliferation and progression of cholangiocarcinoma. ${ }^{56}$ And, TGR5 activation is responsible for bile acid-induced itch and analgesia. ${ }^{57}$

TGR5 is considered as a potential therapeutic target in treatment of cholestasis and metabolic liver diseases; however, further studies are in demand to evaluate long-term therapeutic efficacy and safety.

\section{ASBT}

As mentioned above, ASBT is of vital importance in the enterohepatic circulation of BAs and maintaining the bile acid homeostasis. Inhibition of ASBT results in interruption of bile acid re-absorption and BAs pool shrinkage. A recent study demonstrated that volixibat inhibits bile acid reabsorption via inhibiting ASBT located on the luminal surface of the ileum, which subsequently shows benefit in patients with NASH. ${ }^{58}$ It is suggested that LUM001 (an ASBT inhibitor) reduces the circulating BAs pool via interrupting the reabsorption of BAs, which consequently improves intrahepatic cholestasis. ${ }^{59}$

The ASBT inhibitor SC-435 significantly reduces BAs pool size and retention of hydrophobic BAs, attenuates leukocytes recruitment, and alleviates progression of sclerosing cholangitis in animal models. ${ }^{60}$ Baghdasaryan et al. ${ }^{61}$ suggested that inhibition of intestinal bile acid absorption with A4250, a selective ASBT inhibitor, might improve cholestatic liver injury in sclerosing cholangitis. Besides, BAs have been considered as potential pruritogens in cholestasis. ASBT inhibitors might alleviate pruritus by reducing circulating BAs. In PBC patients with pruritus, it has been demonstrated that the ASBT inhibitor GSK2330672 treatment is effective in alleviating pruritus. ${ }^{62}$

\section{Conclusions}

BAs are natural detergents that play important roles in absorption and utilization of lipid. Accumulation of BAs 
within hepatocytes actually results in cholestatic liver damage. BAs are important signaling molecules, regulating multiple metabolic pathways, including lipid, glucose, drug and energy metabolism. As such, BAs have been considered as therapeutic targets for treatment of cholestatic and metabolic liver diseases. We have discussed herein the emerging therapeutic roles of bile acid signaling in chronic liver diseases. New therapeutic agents were also discussed and the further investigation required for efficacy and safety evaluations. It is anticipated that bile acid signaling will give birth to more inspiration in the near future.

\section{Conflict of interest}

The authors have no conflict of interests related to this publication.

\section{Author contributions}

Wrote the review $(\mathrm{YL})$, edited the review (LGL).

\section{References}

[1] Perez MJ, Briz O. Bile-acid-induced cell injury and protection. World ] Gastroenterol 2009;15:1677-1689. doi: 10.3748/wjg.15.1677.

[2] Chiang JYL, Ferrell JM. Bile acid metabolism in liver pathobiology. Gene Expr 2018;18:71-87. doi: 10.3727/105221618X15156018385515.

[3] Manley S, Ding W. Role of farnesoid X receptor and bile acids in alcoholic liver disease. Acta Pharm Sin B 2015;5:158-167. doi: 10.1016/j.apsb.2014.12. 011.

[4] Ferslew BC, Xie G, Johnston CK, Su M, Stewart PW, Jia W, et al. Altered bile acid metabolome in patients with nonalcoholic steatohepatitis. Dig Dis Sci 2015;60:3318-3328. doi: 10.1007/s10620-015-3776-8

[5] Hegade VS, Speight RA, Etherington RE, Jones DE. Novel bile acid therapeutics for the treatment of chronic liver diseases. Therap Adv Gastroenterol 2016;9:376-391. doi: 10.1177/1756283X16630712.

[6] Klaassen CD, Cui JY. Review: Mechanisms of how the intestinal microbiota alters the effects of drugs and bile acids. Drug Metab Dispos 2015;43:15051521. doi: 10.1124/dmd.115.065698.

[7] Zollner G, Trauner M. Mechanisms of cholestasis. Clin Liver Dis 2008;12: 1-26. doi: 10.1016/j.cld.2007.11.010.

[8] Trauner M, Fuchs CD, Halilbasic E, Paumgartner G. New therapeutic concepts in bile acid transport and signaling for management of cholestasis. Hepatology 2017;65:1393-1404. doi: 10.1002/hep.28991.

[9] Chiang JY. Bile acids: regulation of synthesis. J Lipid Res 2009;50:19551966. doi: 10.1194/jlr.R900010-JLR200.

[10] Baker DM, Wang SL, Bell DJ, Drevon CA, Davis RA. One or more labile proteins regulate the stability of chimeric mRNAs containing the $3^{\prime}$-untranslated region of cholesterol-7alpha -hydroxylase mRNA. J Biol Chem 2000;275: 19985-19991. doi: 10.1074/jbc.M002351200.

[11] Li T, Jahan A, Chiang JY. Bile acids and cytokines inhibit the human cholesterol 7 alpha-hydroxylase gene via the JNK/C-jun pathway in human liver cells. Hepatology 2006;43:1202-1210. doi: 10.1002/hep.21183.

[12] Song KH, Li T, Owsley E, Strom S, Chiang JY. Bile acids activate fibroblast growth factor 19 signaling in human hepatocytes to inhibit cholesterol 7alpha-hydroxylase gene expression. Hepatology 2009;49:297-305. doi: 10.1002/hep.22627.

[13] Holt JA, Luo G, Billin AN, Bisi J, McNeill YY, Kozarsky KF, et al. Definition of a novel growth factor-dependent signal cascade for the suppression of bile acid biosynthesis. Genes Dev 2003;17:1581-1591. doi: 10.1101/gad.1083503.

[14] Sanyal S, Båvner A, Haroniti A, Nilsson LM, Lundåsen T, Rehnmark S, et al. Involvement of corepressor complex subunit GPS2 in transcriptional pathways governing human bile acid biosynthesis. Proc Natl Acad Sci U S A 2007 ; 104:15665-15670. doi: 10.1073/pnas.0706736104.

[15] Chiang JYL. Bile acid metabolism and signaling in liver disease and therapy. Liver Res 2017;1:3-9. doi: 10.1016/j.livres.2017.05.001.

[16] Li T, Kong X, Owsley E, Ellis E, Strom S, Chiang JY. Insulin regulation of cholesterol 7alpha-hydroxylase expression in human hepatocytes: roles of forkhead box $\mathrm{O} 1$ and sterol regulatory element-binding protein 1c. J Biol Chem 2006;281:28745-28754. doi: 10.1074/jbc.M605815200.

[17] Song KH, Chiang JY. Glucagon and cAMP inhibit cholesterol 7alpha-hydroxylase (CYP7A1) gene expression in human hepatocytes: discordant regulation of bile acid synthesis and gluconeogenesis. Hepatology 2006;43:117-125. doi: 10. 1002/hep.20919.

[18] Gälman C, Angelin B, Rudling M. Bile acid synthesis in humans has a rapid diurnal variation that is asynchronous with cholesterol synthesis. Gastroenterology 2005;129:1445-1453. doi: 10.1053/j.gastro.2005.09.009.

[19] Brenner DA, Paik YH, Schnabl B. Role of gut microbiota in liver disease. J Clin Gastroenterol 2015;49 Suppl 1:S25-S27. doi: 10.1097/MCG. 0000000000000391

[20] Ridlon JM, Kang DJ, Hylemon PB. Bile salt biotransformations by human intestinal bacteria. J Lipid Res 2006;47:241-259. doi: 10.1194/jlr. R500013-JLR200.

[21] Ridlon JM, Alves JM, Hylemon PB, Bajaj JS. Cirrhosis, bile acids and gut microbiota: unraveling a complex relationship. Gut Microbes 2013;4:382387. doi: 10.4161/gmic. 25723 .

[22] Phelan JP, Reen FJ, Caparros-Martin JA, O'Connor R, O'Gara F. Rethinking the bile acid/gut microbiome axis in cancer. Oncotarget 2017;8:115736115747. doi: 10.18632/oncotarget.22803.

[23] Bonde Y, Breuer O, Lütjohann D, Sjöberg S, Angelin B, Rudling M. Thyroid hormone reduces PCSK9 and stimulates bile acid synthesis in humans. J Lipid Res 2014;55:2408-2415. doi: 10.1194/jlr.M051664.

[24] Song Y, Xu C, Shao S, Liu J, Xing W, Xu J, et al. Thyroid-stimulating hormone regulates hepatic bile acid homeostasis via SREBP-2/HNF-4 $\alpha /$ CYP7A1 axis. J Hepatol 2015;62:1171-1179. doi: 10.1016/j.jhep.2014.12.006.

[25] Beuers U, Trauner M, Jansen P, Poupon R. New paradigms in the treatment of hepatic cholestasis: from UDCA to FXR, PXR and beyond. J Hepatol 2015;62: S25-S37. doi: 10.1016/j.jhep.2015.02.023.

[26] Hohenester S, Wenniger LM, Paulusma CC, van Vliet SJ, Jefferson DM, Elferink RP, et al. A biliary $\mathrm{HCO} 3-$ umbrella constitutes a protective mechanism against bile acid-induced injury in human cholangiocytes. Hepatology 2012;55:173-183. doi: 10.1002/hep.24691.

[27] Rudic JS, Poropat G, Krstic MN, Bjelakovic G, Gluud C. Ursodeoxycholic acid for primary biliary cirrhosis. Cochrane Database Syst Rev 2012;12: CD000551. doi: 10.1002/14651858.CD000551.pub3.

[28] Zhu GQ, Shi KQ, Huang S, Huang GQ, Lin YQ, Zhou ZR, et al. Network metaanalysis of randomized controlled trials: efficacy and safety of UDCA-based therapies in primary biliary cirrhosis. Medicine (Baltimore) 2015;94:e609. doi: 10.1097/MD.0000000000000609.

[29] Bosch A, Dumortier J, Maucort-Boulch D, Scoazec JY, Wendum D, Conti F, et al. Preventive administration of UDCA after liver transplantation for primary biliary cirrhosis is associated with a lower risk of disease recurrence. J Hepatol 2015;63:1449-1458. doi: 10.1016/j.jhep.2015.07.038.

[30] Chapman R, Fevery J, Kalloo A, Nagorney DM, Boberg KM, Shneider B, et al. Diagnosis and management of primary sclerosing cholangitis. Hepatology 2010;51:660-678. doi: 10.1002/hep.23294.

[31] Zhu GQ, Shi KQ, Huang GQ, Wang LR, Lin YQ, Braddock M, et al. A network meta-analysis of the efficacy and side effects of UDCA-based therapies for primary sclerosing cholangitis. Oncotarget 2015;6:26757-26769. doi: 10. 18632/oncotarget.5610.

[32] Munoz-Garrido P, Marin J], Perugorria MJ, Urribarri AD, Erice O, Sáez E, et al. Ursodeoxycholic acid inhibits hepatic cystogenesis in experimental models of polycystic liver disease. J Hepatol 2015;63:952-961. doi: 10.1016/j.jhep. 2015.05.023.

[33] Perugorria MJ, Labiano I, Esparza-Baquer A, Marzioni M, Marin JJ, Bujanda L, et al. Bile acids in polycystic liver diseases: Triggers of disease progression and potential solution for treatment. Dig Dis 2017;35:275-281. doi: 10 . $1159 / 000450989$.

[34] Halilbasic E, Steinacher D, Trauner M. Nor-ursodeoxycholic acid as a novel therapeutic approach for cholestatic and metabolic liver diseases. Dig Dis 2017;35:288-292. doi: 10.1159/000454904.

[35] Krones E, Eller K, Pollheimer MJ, Racedo S, Kirsch AH, Frauscher B, et al. NorUrsodeoxycholic acid ameliorates cholemic nephropathy in bile duct ligated mice. J Hepatol 2017;67:110-119. doi: 10.1016/j.jhep.2017.02.019.

[36] Fickert P, Hirschfield GM, Denk G, Marschall HU, Altorjay I, Färkkilä M, et al. norUrsodeoxycholic acid improves cholestasis in primary sclerosing cholangitis. J Hepatol 2017;67:549-558. doi: 10.1016/j.jhep.2017.05.009.

[37] Fickert P, Pollheimer MJ, Silbert D, Moustafa T, Halilbasic E, Krones E, et al. Differential effects of norUDCA and UDCA in obstructive cholestasis in mice. J Hepatol 2013;58:1201-1208. doi: 10.1016/j.jhep.2013.01.026.

[38] Sombetzki M, Fuchs CD, Fickert $\mathrm{P}$, Österreicher $\mathrm{CH}$, Mueller $\mathrm{M}$, Claudel $\mathrm{T}$, et al. 24-nor-ursodeoxycholic acid ameliorates inflammatory response and liver fibrosis in a murine model of hepatic schistosomiasis. J Hepatol 2015; 62:871-878. doi: 10.1016/j.jhep.2014.11.020.

[39] Copple BL, Li T. Pharmacology of bile acid receptors: Evolution of bile acids from simple detergents to complex signaling molecules. Pharmacol Res 2016;104:9-21. doi: 10.1016/j.phrs.2015.12.007

[40] Adorini L, Pruzanski M, Shapiro D. Farnesoid X receptor targeting to treat nonalcoholic steatohepatitis. Drug Discov Today 2012;17:988-997. doi: 10.1016/j.drudis.2012.05.012.

[41] Mudaliar S, Henry RR, Sanyal AJ, Morrow L, Marschall HU, Kipnes M, et al. Efficacy and safety of the farnesoid $X$ receptor agonist obeticholic acid in 
patients with type 2 diabetes and nonalcoholic fatty liver disease. Gastroenterology 2013;145:574-582.e1. doi: 10.1053/j.gastro.2013.05.042.

[42] Jouihan H, Will S, Guionaud S, Boland ML, Oldham S, Ravn P, et al. Superior reductions in hepatic steatosis and fibrosis with co-administration of a glucagon-like peptide-1 receptor agonist and obeticholic acid in mice. Mol Metab 2017;6:1360-1370. doi: 10.1016/j.molmet. 2017. 09.001 .

[43] Verbeke L, Farre R, Trebicka J, Komuta M, Roskams T, Klein S, et al. Obeticholic acid, a farnesoid $\mathrm{X}$ receptor agonist, improves portal hypertension by two distinct pathways in cirrhotic rats. Hepatology 2014;59:2286-2298. doi: 10.1002/hep.26939.

[44] Schwabl P, Hambruch E, Seeland BA, Hayden H, Wagner M, Garnys L, et al. The FXR agonist PX20606 ameliorates portal hypertension by targeting vascular remodelling and sinusoidal dysfunction. J Hepatol 2017;66:724-733. doi: $10.1016 /$ j.jhep.2016.12.005.

[45] Carino A, Cipriani S, Marchianò S, Biagioli M, Santorelli C, Donini A, et al. BAR502, a dual FXR and GPBAR1 agonist, promotes browning of white adipose tissue and reverses liver steatosis and fibrosis. Sci Rep 2017;7: 42801. doi: $10.1038 /$ srep42801.

[46] Honda A, Ikegami T, Nakamuta M, Miyazaki T, Iwamoto J, Hirayama T, et al. Anticholestatic effects of bezafibrate in patients with primary biliary cirrhosis treated with ursodeoxycholic acid. Hepatology 2013;57:1931-1941. doi: $10.1002 /$ hep. 26018

[47] Cuperus FJ, Halilbasic E, Trauner M. Fibrate treatment for primary biliary cirrhosis. Curr Opin Gastroenterol 2014;30:279-286. doi: 10.1097/MOG. 0000000000000056.

[48] Iwasaki S, Akisawa N, Saibara T, Onishi S. Fibrate for treatment of primary biliary cirrhosis. Hepatol Res 2007;37:S515-S517. doi: 10.1111/j.1872034X.2007.00232.x.

[49] Dohmen K, Mizuta T, Nakamuta M, Shimohashi N, Ishibashi H, Yamamoto K. Fenofibrate for patients with asymptomatic primary biliary cirrhosis. World ] Gastroenterol 2004;10:894-898. doi: 10.3748/wjg.v10.i6.894.

[50] Dai M, Yang J, Xie M, Lin J, Luo M, Hua H, et al. Inhibition of JNK signalling mediates PPAR $\alpha$-dependent protection against intrahepatic cholestasis by fenofibrate. $\mathrm{Br}$ J Pharmacol 2017;174:3000-3017. doi: 10.1111/bph. 13928.

[51] Keitel V, Reich M, Häussinger D. TGR5: pathogenetic role and/or therapeutic target in fibrosing cholangitis? Clin Rev Allergy Immunol 2015;48:218-225. doi: $10.1007 / \mathrm{s} 12016-014-8443-x$.

[52] Malhi H, Camilleri M. Modulating bile acid pathways and TGR5 receptors for treating liver and GI diseases. Curr Opin Pharmacol 2017;37:80-86. doi: 10. 1016/j.coph.2017.09.008.
[53] McMillin M, Frampton G, Tobin R, Dusio G, Smith J, Shin H, et al. TGR5 signaling reduces neuroinflammation during hepatic encephalopathy. J Neurochem 2015;135:565-576. doi: 10.1111/jnc.13243.

[54] De Oliveira MC, Gilglioni EH, De Boer BA, Runge JH, De Waart DR, Salgueiro $\mathrm{CL}$, et al. Bile acid receptor agonists INT747 and INT777 decrease oestrogen deficiency-related postmenopausal obesity and hepatic steatosis in mice. Biochim Biophys Acta 2016;1862:2054-2062. doi: 10.1016/j.bbadis. 2016 . 07.012 .

[55] Rizzo G, Passeri D, De Franco F, Ciaccioli G, Donadio L, Rizzo G, et al. Functional characterization of the semisynthetic bile acid derivative INT-767, dual farnesoid X receptor and TGR5 agonist. Mol Pharmacol 2010;78:617630. doi: $10.1124 / \mathrm{mol} .110 .064501$.

[56] Erice O, Labiano I, Arbelaiz A, Santos-Laso A, Munoz-Garrido P, JimenezAgüero R, et al. Differential effects of FXR or TGR5 activation in cholangio carcinoma progression. Biochim Biophys Acta 2018;1864:1335-1344. doi: 10.1016/j.bbadis.2017.08.016.

[57] Alemi F, Kwon E, Poole DP, Lieu T, Lyo V, Cattaruzza F, et al. The TGR5 receptor mediates bile acid-induced itch and analgesia. J Clin Invest 2013;123: 1513-1530. doi: $10.1172 /$ JCI64551.

[58] Palmer M, Jennings L, Silberg DG, Bliss C, Martin P. A randomised, doubleblind, placebo-controlled phase 1 study of the safety, tolerability and pharmacodynamics of volixibat in overweight and obese but otherwise healthy adults: implications for treatment of non-alcoholic steatohepatitis. BMC Pharmacol Toxicol 2018;19:10. doi: 10.1186/s40360-018-0200-y.

[59] Xiao L, Pan G. An important intestinal transporter that regulates the enterohepatic circulation of bile acids and cholesterol homeostasis: The apical sodium-dependent bile acid transporter (SLC10A2/ASBT). Clin Res Hepato Gastroenterol 2017;41:509-515. doi: 10.1016/j.clinre.2017.02.001.

[60] Miethke AG, Zhang W, Simmons J, Taylor AE, Shi T, Shanmukhappa SK, et al. Pharmacological inhibition of apical sodium-dependent bile acid transporter changes bile composition and blocks progression of sclerosing cholangitis in multidrug resistance 2 knockout mice. Hepatology 2016;63:512-523. doi: 10.1002/hep.27973.

[61] Baghdasaryan A, Fuchs CD, Österreicher $\mathrm{CH}$, Lemberger U], Halilbasic E, Påhlman I, et al. Inhibition of intestinal bile acid absorption improves cholestatic liver and bile duct injury in a mouse model of sclerosing cholangitis. J Hepatol 2016;64:674-681. doi: 10.1016/j.jhep.2015.10.024.

[62] Hegade VS, Kendrick SF, Dobbins RL, Miller SR, Thompson D, Richards D, et al. Effect of ileal bile acid transporter inhibitor GSK2330672 on pruritus in primary biliary cholangitis: a double-blind, randomised, placebo-controlled, crossover, phase $2 a$ study. Lancet 2017;389:1114-1123. doi: 10 . 1016/S0140-6736(17)30319-7. 